\section{Response to Snijders et al.}

doi:10.1038/pr.2014.80

To the Editor: We thank Drs. Snijders, Bertozzi, and Barbato (1) for their interest in our review of advances in the genetics of primary ciliary dyskinesia (PCD) (2). New genetic discoveries have greatly advanced our understanding of the disease but also reveal limitations in the current diagnostic criteria. As we stated in our review, transmission electron microscopy has historically been the cornerstone of PCD diagnosis. Recent descriptions of mutations that produce a clinical phenotype with normal ultrastructure render the use of transmission electron microscopy as a sole modality for PCD diagnosis unacceptable $(3,4)$. Moreover, transmission electron microscopy is technically demanding, expensive, time consuming, and does not exclude secondary causes of abnormal axonemal structure.

Thus, the crux of the matter is to identify alternative diagnostic tests that are accurate (sensitive and specific), highly reproducible, and simple to perform at a center that may see PCD patients. Their comments reflect a divergence between European and NorthAmerican approaches toward diagnostic testing. In Europe, highspeed video microscopy has become the preferred diagnostic test for PCD, which is based on a consensus document from 2009 (5). High-speed video microscopy requires substantial experience and is best performed in centers that specialize in PCD. In this setting, the method can be useful in screening for PCD, particularly in cases with immotile or stiff, dysmotile cilia. However, this approach has significant limitations. For mutations characterized by subtle or variable changes in ciliary waveform, such as those affecting DNAH11 or radial spoke genes, the presence of near normal high-speed video microscopy may lead to a false-negative diagnosis. Alternatively, slow or abnormal beating as occurs after infection or exposure to inhaled irritants can be an acquired defect, and may lead to a false-positive conclusion. Moreover, cilia beat can be affected by the manipulation of a fresh tissue sample, to the point of producing a different phenotype after the retrieved airway cells are allowed to recover in culture, as suggested in a recent report from a European center specializing in PCD (6).

Nasal nitric oxide levels have increasingly been adopted as the diagnostic test for PCD in North-American centers, especially given the improved standardization of measurements (7), thus, avoiding reliance on ultrastructural defects or even cilia beat pattern in select patients. While the test has some limitations (e.g., overlapping levels in individuals with cystic fibrosis), a recent multicenter study of 149 subjects with PCD and 224 healthy and disease controls showed that the technique was safe, easy to perform, highly reproducible, and had sensitivity and specificity of 0.98 and 0.99 , respectively, in patients 5 years of age and older (7). There is growing evidence that nasal nitric oxide levels may even discriminate PCD from other lung diseases in preschool populations (unpublished observations). Until genetic diagnosis is approved, we contend that a validated set of diagnostic criteria should include nasal nitric oxide levels.

\section{Amjad Horani', Steven L. Brody ${ }^{2}$ and Thomas W. Ferkol ${ }^{1,3}$}

${ }^{1}$ Department of Pediatrics, Washington University School of Medicine, St Louis, Missouri; ${ }^{2}$ Department of Medicine, Washington University School of Medicine, St Louis, Missouri; ${ }^{3}$ Department of Cell Biology and Physiology, Washington University School of Medicine, St Louis, Missouri.

Correspondence: Thomas W. Ferkol (ferkol_t@kids.wustl.edu)

\section{REFERENCES}

1. Snijders D, Bertozzi I, Barbato A,Nasal NO. high-speed video microscopy, electron microscopy, and genetics: a primary ciliary dyskinesia puzzle to complete Pediatr Res 2014;76:321.

2. Horani A, Brody SL, Ferkol TW. Picking up speed: advances in the genetics of primary ciliary dyskinesia. Pediatr Res 2014;75:158-64.

3. Knowles MR, Leigh MW, Carson JL, et al.; Genetic Disorders of Mucociliary Clearance Consortium. Mutations of DNAH11 in patients with primary ciliary dyskinesia with normal ciliary ultrastructure. Thorax 2012;67:433-41.

4. Horani A, Brody SL, Ferkol TW, et al. CCDC65 mutation causes primary ciliary dyskinesia with normal ultrastructure and hyperkinetic cilia. PLoS One 2013;8:e72299.

5. Barbato A, Frischer T, Kuehni CE, et al. Primary ciliary dyskinesia: a consensus statement on diagnostic and treatment approaches in children. Eur Respir J 2009;34:1264-76.

6. Boon M, Smits A, Cuppens H, et al. Primary ciliary dyskinesia: critical evaluation of clinical symptoms and diagnosis in patients with normal and abnormal ultrastructure. Orphanet J Rare Dis 2014;9:11.

7. Leigh MW, Hazucha MJ, Chawla KK, et al. Standardizing nasal nitric oxide measurement as a test for primary ciliary dyskinesia. Ann Am Thorac Soc 2013;10:574-81. 